were $0.82(0.73,0.90)$ and $0.99(0.89,1.08)$ respectively. Responses were consistent across vignette gender and most respondent characteristics. However, the relative importance given to different dimensions varied with respondent's age. Differences for social engagement remained fairly constant at all ages, while the relative importance of disease decreased somewhat with increasing age. In contrast, differences for physical function, cognitive function, and productive engagement increased with increasing age.

Conclusion Clinical definitions of successful ageing that focus on longevity and disease do not reflect the views of the general population. In order to support and promote successful ageing, practitioners and policy makers should be aware of older people's priorities for ageing and, in particular, understand how these differ from their own.

\section{OP83 \#IS VOLUME OF PHYSICAL ACTIVITY MORE IMPORTANT THAN PATTERN OF ACCUMULATION FOR ONSET OF CARDIOVASCULAR DISEASE? A PROSPECTIVE STUDY OF OBJECTIVELY MEASURED PHYSICAL ACTIVITY INTENSITIES AND SEDENTARY BEHAVIOUR IN OLDER MEN}

1,2BJ Jefferis* 1,2TJ Parsons, 1,2 C Sartini, 1,2S Ash, 1'LT Lennon, ${ }^{1} 0$ Papacosta, ${ }^{1} \mathrm{SG}$ Wannamethee, ${ }^{3} \mathrm{IM}$ Lee, ${ }^{4} \mathrm{PH}$ Whincup. ${ }^{1}$ Primary Care and Population Health, University College London, London, UK; ${ }^{2}$ Physical Activity Research Group, University College London, London, UK; ${ }^{3}$ Harvard Medical School, Brigham and Women's Hospital, Boston, USA; ${ }^{4}$ Population Health Research Institute, St George's University of London, London, UK

\subsection{6/jech-2018-SSMabstracts.82}

Aim To understand how device-measured sedentary behaviour and physical activity are related to cardiovascular disease (CVD) events in older men, an age-group with high levels of physical inactivity and sedentary behaviour. Activity monitors permit investigation of different activity intensities, (including light activity which is hard to recall) and the pattern of accumulating activity, (such as doing moderate to vigorous physical activity (MVPA) in $10 \mathrm{~min}$ bouts) or breaking up periods of sedentary behaviour.

Methods Prospective population-based cohort study of 7735 men recruited from 24 UK General Practices in 1978-80. In 2010-12, 3137 surviving men were invited to complete a questionnaire about medical history and health behaviours and to wear an Actigraph GT3x accelerometer over the hip for 7 days. Physical activity intensity was categorised as sedentary: <100 counts/minute, light: 100-1040 counts/minute and moderate to vigorous PA (MVPA): >1040 counts/minute. A sedentary break was the interruption of a sedentary bout lasting $>1 \mathrm{~min}$ by $\geq 1 \mathrm{~min}$ of activity $>100$ counts/minute. Men were followed up for CVD morbidity (ICD9 410-414 and 430-438) and mortality from 2010-12 to 1 st June 2016. Cox proportional hazards models estimated Hazard Ratios (HRs) for CVD according to physical activity measured in 2010-12, controlling for confounders.

Results 1,566/3137 (50\%) men returned an accelerometer with data and 1528 (49\%) had $\geq 600$ minutes/day wear time on $\geq 3$ days. 254 men with pre-existing CVD were excluded, leaving 1274 men. Participants' mean age was 78.4 (range 7192) years. After median 4.6 years follow-up, 82 first CVD events occurred in 1181 men with complete covariate data. For each additional $30 \mathrm{~min}$ in sedentary behaviour, light physical activity, $10 \mathrm{~min}$ in MVPA, or 1000 steps/day, hazard ratios for CVD mortality were 1.08 (95\% CI 0.98, 1.18), 0.96
(95\% CI $0.85,1.08), 0.89$ (95\% CI $0.81,0.98)$ and 0.86 (95\% CI 0.77 to 0.96 ) respectively. Adjustments for confounders little changed the estimates. The hazard ratio for accumulating $150 \mathrm{~min} \mathrm{MVPA} /$ week irrespective of bout length (achieved by $66 \%$ of men) was 0.54 (95\% CI, 0.34 to 0.87 ) and for accumulating $150 \mathrm{~min} \mathrm{MVPA} /$ week in bouts lasting $\geq 10 \mathrm{~min}$ (achieved by $16 \%$ of men) was $0.49(95 \%$ CI, 0.21 to 1.13$)$. Sedentary breaks were not associated with CVD events.

Discussion In older men, a higher number of steps per day or accumulating more MVPA (irrespective of bouts lasting $\geq 10 \mathrm{~min}$ ) was associated with reduced CVD risk. Hence, in this population CVD prevention could focus on accumulating time in activities like brisk walking each day. Future studies should replicate analyses in women and younger populations.

\section{OP84 IS SOCIAL DISADVANTAGE A CHRONIC STRESSOR? SOCIOECONOMIC POSITION AND CHRONIC STRESS AMONG OLDER ADULTS LIVING IN ENGLAND}

G Chatzi ${ }^{\star}$, T Chandola, A Cernat, N Shlomo. Social Statistics, University of Manchester, Manchester, UK

\subsection{6/jech-2018-SSMabstracts.83}

Background Living in social disadvantage has been conceptualised as a chronic stressor, although this contradicts evidence from recent studies using hair cortisol as a measure of hypothalamus-pituitary-adrenal (HPA) axis activity. The methodological limitations of previous studies investigating the association between socioeconomic position (SEP) and hair cortisol and cortisone are taken into account in this study which examines if lower SEP is associated with higher levels of HPA axis activity as measured by hair cortisol and cortisone among older adults.

Methods Cortisol and cortisone levels in hair samples from 2468 participants in the 6th wave of the English Longitudinal Study of Ageing (ELSA) are examined, in relation to educational attainment, wealth, social class, and employment status. Multivariable linear regression models were used to examine the association between socioeconomic position and cortisol and cortisone levels. Inverse probability weighting and multiple imputation were used to compensate for missing data. Interactions between social class and employment status were tested. All models were adjusted for gender, age, interaction between gender and age, ethnicity, marital status, hair treatment, hair colour, nurse visiting month, smoking status, body mass index, self-assessed health, number of medications, and depressive symptoms.

Results We found significant differences between the most and least advantaged social classes in their levels of hair cortisol and cortisone. Participants in the lower supervisory social class and retired had increased levels of cortisol $(0.71 \log (\mathrm{pg} / \mathrm{mg})$, 95\% CI 0.14 to 1.28$)$ and cortisone $(0.73 \log (\mathrm{pg} / \mathrm{mg}), 95 \% \mathrm{CI}$ 0.29 to 1.16 ) compared to participants in the most advantaged social class and those still in work. Among the economically inactive, the most disadvantaged social classes clearly had increased levels of hair cortisol and cortisone. Further analyses that take missing data into account showed that the complete case estimates of hair cortisone in the most disadvantaged groups were underestimated compared to estimates accounting for missing data, such as inverse probability weighting and multiple imputation. 